\title{
Laser Ultrasonic Porcelain Insulator According to Simulation Studies
}

\author{
Min $\mathrm{Ji}^{1,}$ a and Pengfei $\mathrm{Ji}^{2, \mathrm{~b}}$ \\ ${ }^{1}$ Xijing University, Shaanxi Xi'an, 710123, China \\ ${ }^{2}$ China Railway 21 Bureau Group Second Engineering Co. Ltd., Shaanxi, xi 'an 710065, China \\ a995010771@qq.com,b573825625@qq.com
}

Keywords: Laser ultrasonic; Temperature; Vibration

\begin{abstract}
This paper first summarizes the laser ultrasonic according to material excitation mechanism of ultrasonic, starting from the thermo elastic mechanism of pulse excitation laser ultrasonic, laser line source as the excitation light source, the thermoelastic mechanism under pulsed laser $\mathrm{f}$ as porcelain insulator material on the transient temperature field and displacement field of the finite element numerical simulation. The laser transmission power source too, porcelain insulator surface distribution of transient temperature field and thermal stress caused by the size of the vibration displacement amplitude and changing trends.
\end{abstract}

\section{Introduction}

Laser ultrasonic refers to the laser light source f objects and interact with the object of ultrasound, the blessing of laser ultrasound mainly depends on the performance of laser light source according to the pattern, the modulation of the light beam of light absorption performance and the material surface. Laser governs as material to produce ultrasonic radiation pressure incentive mechanism, the mechanism of electrostrictive mechanism, mechanism of dielectric breakdown, vaporizing expansion mechanism, thermoelastic mechanism and the ablation mechanism according to the incident laser power and the material being measured melting candle threshold, laser pulse excitation mechanism of ultrasonic wave can be divided into two kinds, namely the laser ultrasonic thermoelastic mechanism and absorption mechanism. Among them, the thermoelastic mechanism of ultrasound on the test itself has no damage, can be used in the nondestructive testing in various fields.

\section{The Laser Ultrasonic Hot Spring Mechanism}

When the pulse laser source for less than the power density of the according to the surface of a solid material, according to different material absorption coefficient and the reflection coefficient, the part of the incident energy absorbed by the object, part is reflected in the material surface. Due to the thermal diffusion effect on the surface of the solid material and the effect of material heat bilges cold shrink, will produce certain thermal stress in the material surface area, thus turning light energy into acoustic energy, the corresponding stress wave inside the object. Will this due to the material to absorb light energy (heat) thermal elastic expansion of ultrasonic excitation is called the thermoelastic mechanism of laser ultrasound. Thermoelastic mechanism under the material to absorb sunlight, as a result of the laser pulse, thermal diffusion is very small, only make the material surface within the scope of the depth of nanoscale produce a dozens to hundreds of temperature rise. Blessed by the pulse laser can be tested as a formation of the thermal stress in the material surface area as a surface layer of the transient heat source, and it can be seen as a heat transfer process of transient thermal strain and transient thermal stress formed under the action of the principal stress parallel to the surface of elastic waves.

\section{The Laser Ultrasonic Melt Candle}

Due to the laser power density is bigger, makes the solid material in the suction Collect sunlight $\mathrm{f}$ according to the material surface temperature has risen sharply, in a very short 
period of time than governs according to the melting point of material, and in according to solid material surface under several microns deep areas of candle burning, because evaporation in governs according to solid material surface to form a small amount of plasma. At this point, the laser $f$ getting there will be a small part of the surface material is ejected out, similar to $\mathrm{f}$ according to solid material by pulse laser has imposed a recoil force perpendicular to the surface, thus producing ultrasonic, the laser ultrasonic excitation is sometimes called melting candle mechanism.

\section{The Laser Ultrasonic According to Numerical Simulation of Porcelain Insulator}

With single pulse laser as the excitation source load to the surface of porcelain insulator, the finite element method for the transient temperature field and displacement field of numerical simulation. Simulation analysis, ignore the material light penetration effect, thermal diffusion effect and the influence of temperature change on the material physical parameters. Set the initial temperature of room temperature $\mathrm{T} 0=300 \mathrm{k}$, single pulse of the laser pulse width of $10 \mathrm{~ns}$, energy is $200 \mathrm{mj}$, spot radius of $0.5 \mathrm{~mm}$, at 1064 .Considering for the insulator, thermal transfer only in a small range, simulate the insulator only an umbrella group of scope for analysis. Measured, its height is $1.8 \mathrm{~cm}$, inner cylinder radius of $3.2 \mathrm{~cm}$, an umbrella group of radius of $5 \mathrm{~cm}$, an umbrella group of thickness is $1 \mathrm{~cm}$.

Fig. 1 is the circumferential distribution along the external surface of the porcelain insulator model graph. The graph curve relationship shows that the laser on the insulator model of transient temperature field are mainly distributed in the laser heat source near the $1.382 \mathrm{~mm}$, and with the distances from the center of the laser heat source model of the node temperature decreases with the increasing of distance. When nodes away from heat source center is less than $0.5 \mathrm{~mm}$ range, temperature from $545 \mathrm{k}$ to $350 \mathrm{k}$ quickly; After that, the temperature changing with distance down gently. Laser heating source range is very narrow.

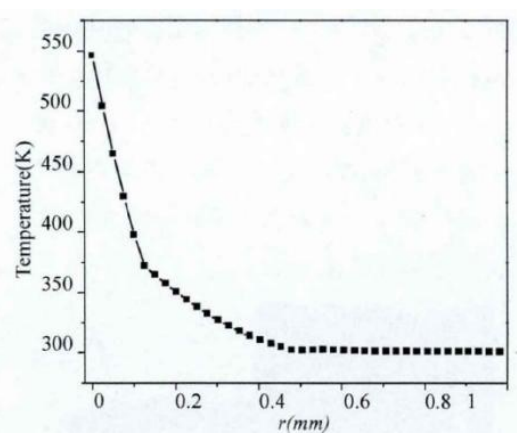

Figure 1. figure circumferential temperature temperature distribution distribution curve

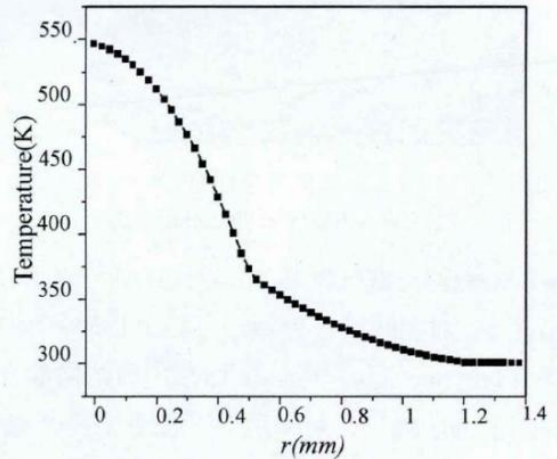

Figure 2. radial curve

Fig.2 is a porcelain insulator model along the radial temperature distribution curve, the curve in figure relationship, the model of the radial temperature range is $0.959 \mathrm{~mm}$, weeks to a more severe degree of temperature with the decline of distance; show that laser heat source decay quickly in the depth direction. That is to say, the laser can only be shallow depth range of heating on the surface of a structure. In a word, along the path of circumferential and radial node temperature is different, the rate of change of temperature or interval in larger circumferential temperature range. 


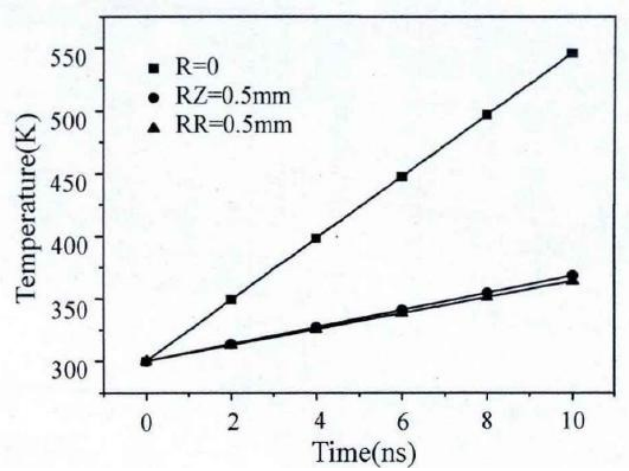

Figure 3. different positions temperature change curve

Fig. 3 is porcelain insulator model different nodes with duration of laser heat source temperature change curve. Said in its R0 $=0$ of the center of the laser line source node temperature time curve, $\mathrm{RZ}=0.5 \mathrm{~mm}$ said circumferential direction of $0.5 \mathrm{~mm}$ distances from the center of the laser line source node temperature time curve, RR said radial direction distances from the center of the laser line source $0.5 \mathrm{~mm}$ node temperature time curve.

By Fig.3 curve relationship, the laser heat source f photo, outside surface absorption of laser energy and translated into its own heat energy, the laser on the porcelain insulator model according to surrounding temperature gradually raised, eventually forming local temperature field.

When the laser heat source, the sample due to the change of the transient temperature field and the stress field near the heat source, which, under the action of stress field, different particle vibration displacement produced by the receiver. As shown in Fig.4, A, B, C, D, four points location diagram of particle displacement and time. The Fig. 4 shows that under the action of pulse laser source, particle vibration to do a small value. Point A receiver, the large amplitude around 15nm; B receives less points out the surface displacement of detected signal, the amplitude is $1.5 \mathrm{~nm}$, point $\mathrm{C}$ receive smaller displacement. Visible, light source, the more distant distance, the smaller the particle vibration amplitude.
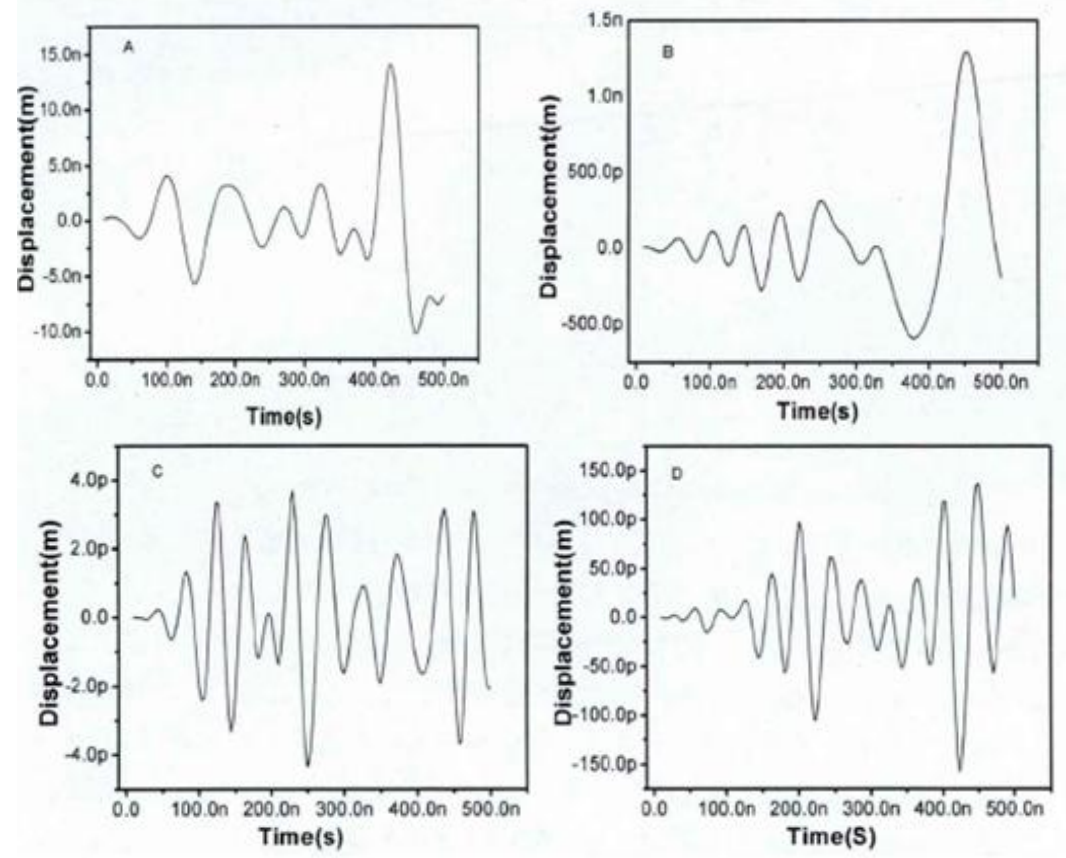

Figure 4. different receiver particle vibration displacement time curve

\section{Summary}

Finite element numerical simulation results show that the pulsed laser $\mathrm{f}$ as porcelain insulator material incentive ultrasonic displacement signal is small, its vibration displacement in $\mathrm{nm}$ node, usually around between several nanometers to a dozen. With the laser ultrasonic photoacoustic 
conversion rate lower theoretical basis is consistent, but also for the node of vibration displacement precision of weak signal detection put forward high request, that is let hair can detect weak acoustic signal of high precision detection method, in order to improve the sensitivity of the laser ultrasonic nondestructive testing.

\section{References}

[1] H. Yamawaki and T. Saito, "Numerical calculation of ultrasonic propagation with anisotropy", NDT \& E Int., Vol.33, Issue7, 2012, pp489-497.

[2] BAHADUR S, Badruddin R. Erosion particle characterization and the effect of particle size and shape on erosion [J]. Wear, 1990 (138): 1892208.

[3] B. Wang, J. Takatsubo, et al., "An Improved Ray Tracing Algorithm for Ultrasonic CT in Nondestructive Inspections", Proceedings of the 8th international Conference on Signal Processing, Vol.1, pp5-8. Nov. 2016 in Guilin, CHINA. 